\title{
Tratamiento conservador de las fracturas del cóndilo mandibular en pacientes pediátricos: Serie de casos.
}

\section{Resumen}

La fractura del cóndilo mandibular es una lesión traumática cuya prevalencia en niños es relativamente baja, lo cual puede deberse tanto a factores anatómicos como ambientales. Las consecuencias incluyen anquilosis de la articulación temporomandibular, asimetrías faciales y trastornos funcionales. El tratamiento conservador es el que cuenta con mayor aceptación, siendo el seguimiento cercano y a largo plazo fundamental. Esta investigación describe el seguimiento de 11 pacientes con antecedentes de fracturas condilares, que asistieron al servicio de Ortodoncia Interceptiva de la Facultad de Odontología de la UCV durante el periodo comprendido entre 2001-2014. Se evaluó la edad del paciente, causa de la fractura, el tipo de fractura, tratamiento recibido y tiempo de duración mediante radiografías panorámicas y tomografías antes y después del tratamiento
Ana Verónica D'Andrea ${ }^{1}$;

A Carolina Medina ${ }^{2}$;

María Gabriela Martínez ${ }^{3}$;

Luzia Da Silva ${ }^{4}$; conservador. Se evaluaron los cambios anatómicos ocurridos. Los tipos de tratamientos variaron desde uso de analgésicos, antiinflamatorios, fisioterapia (ejercicios de apertura y cierre mediante abrebocas y paletas de mordida), aparatos funcionales (Bionator, Klammt, híbridos), y fijación intermaxilar. La prevalencia de fracturas condilares fue de 1,50\% siendo las causas más frecuentes caídas de altura y accidentes de tránsito. Las fracturas mas prevalentes fueron 5 casos de fracturas unilaterales y 6 casos de fracturas bilaterales. El tratamiento conservador resultó exitoso en la vasta mayoría de los casos. Como resultado terapéutico se observó remodelado del cóndilo, en ocasiones con leves variantes anatómicas, simetría facial en reposo y apertura. Ningún caso presentó anquilosis de la ATM.

Palabras Clave: Fractura del cóndilo mandibular; tratamiento conservador.

\footnotetext{
${ }^{1}$ Especialista en Odontología Infantil. Universidad Central de Venezuela.

${ }^{2}$ Especialista en Odontología Infantil, MSc en Odontología, Profesor Titular, Departamento de Odontopediatría y Ortodoncia, Facultad de Odontología, UCV.

${ }^{3}$ Especialista en Odontología Infantil, Profesor Asistente, Coordinador del Programa de Especialización en Odontopediatría, UCV.

${ }^{4}$ Especialista en Odontología Infantil. Especialista en Ortodoncia, Profesor Titular, Departamento de Odontopediatría y Ortodoncia, Facultad de Odontología, UCV.
} 
Relato de caso

\section{Tratamento conservador das fraturas de côndilo mandibular em crianças: Relatório de casos.}

\section{Resumo}

A fratura do côndilo mandibular é uma lesão traumática infrequente em crianças devido a fatores anatômicos e ambientais, além de causar anquilose da articulação temporomandibular, assimetrias faciais e transtornos funcionais. O tratamento conservador é o mais aceito e é fundamental o acompanhamento em curto e longo prazo. A seguinte pesquisa descreve o acompanhamento de 11 pacientes que apresentaram fraturas de côndilo préviamente e visitaram o consultório de Ortodontia Interceptativa da Faculdade de Ontologia da Universidade Central da Venezuela no período 2001-2014. Utilizaram-se radiografia panorâmicas e tomografias antes e depois o tratamento para avaliar a idade do paciente, tipo e causa da fratura, tratamento realizado e o tem- po de duração do acompanhamento. Avaliaram-se as mudanças anatômicas acontecidas. Os tipos de tratamento incluíram analgésicos, anti-inflamatórios, bloqueio intermaxilar, fisioterapia e aparelhos funcionais (bionator, klammt, híbridos). A presença de fraturas de côndilo foi de $1,50 \%$ e as causas mais frequentes foram quedas e acidente de tráfego. As fraturas mais comuns foram 5 casos de fraturas unilaterais e 6 de fraturas bilaterais. $\mathrm{O}$ tratamento conservador foi sucesso na maior parte dos casos. Observou-se como resultado a remodelação do côndilo com variantes anatômicas menores nalgumas ocasiões. Não se observou nem anquilose nem assimetria facial ou funcional.

Palavras chave: Fratura de côndilo mandibular; Tratamento conservador

\section{Conservative treatment of mandibular condyle fractures in children: Case series.}

\begin{abstract}
Fractures of the mandibular condyle are infrequent in children, due to morphologic and other factors. Consequences may include ankylosis of the temporomandibular joint, facial asymmetry and functional disturbances. Conservative treatment is widely accepted, being that close long-term follow-up is fundamental. This report
\end{abstract}

includes 11 cases of children with condylar fractures who were treated at the Interceptive Orthodontics Clinic, Universidad Central de Venezuela Dental School, between 2001 and 2014. Evaluated factors include: age, cause and type of the fracture, treatment performed and follow-up period; which were studied using clinical records: panoramic radiographs, tomograms and photographs before and after treatment. Treatment 
performed included analgesic, anti-inflammatory drugs, intermaxillary fixation, physiotherapy and functional appliances (hybrids, Klammt, Bionator). Prevalence was 1,50\% with falls from heights and traffic accidents the most common cause. Most prevalent fractures were 5 cases of unilateral fractures and 6 cases of bilateral fractures. Conservative treatment was successful in the majority of cases. For most cases satisfactory remodeling of the condyle was attained, with some morphologic variations; no ankylosis, facial asymmetry or functional anomalies were observed after treatment.

Key words: Fractures of the mandibular condyle; conservative treatment.

\section{Introducción}

La fractura del cóndilo mandibular es una lesión traumática que puede producir una discontinuidad de las estructuras adyacentes, acompañado por alteraciones en el rango normal de las excursiones mandibulares. ${ }^{1}$

Los traumatismos faciales y bucales ocurren con frecuencia en la infancia, con una prevalencia entre $28 \%$ y $60 \%$, siendo las fracturas mandibulares las más frecuentes de los huesos faciales en pacientes pediátricos. Dentro de las fracturas mandibulares, las fracturas del cóndilo representan más del 50\% en niños, siendo el cóndilo el sitio más frágil y vulnerable de la mandíbula. ${ }^{2-5}$

Aunque el cóndilo mandibular se encuentra muy protegido frente a los traumatismos directos, su fractura suele producirse de forma indirecta y habitualmente es secundaria a golpes en el mentón. ${ }^{6}$

La causa principal de las fracturas condilares varia significativamente de una sociedad a otra. Amara- tunga $^{7}$ determinó que las fracturas condilares en los niños en Sri Lanka eran diferentes con respecto a los países occidentales, encontrando que la causa más común eran las caídas de altura, seguida por los accidentes de tránsito. Sin embargo en los Estados Unidos, Reino Unido y Europa Occidental la causa principal fueron accidentes de tránsito. En los países bajos, donde montar bicicleta es una forma común de transporte, los accidentes de ciclismo son la causa principal de lesiones condilares. Dimitroulis ${ }^{8}$ sugiere que la etiología está relacionada con la edad y con la actividad que mas desempeñan los pacientes.

Sin embargo, las consecuencias sobre el crecimiento y desarrollo craneofacial que pueden derivar de estas fracturas son de especial importancia, que pueden ir desde anquilosis de la articulación témporomandibular, asimetrías faciales hasta trastornos funcionales. Este tipo de consecuencias deben ser prevenidas por medio de la implementación de una terapia apropiada, según el tipo de fractura. ${ }^{14}$

Las opciones de tratamiento que se pueden emplear en las fracturas del cóndilo mandibular son: reducción cerrada (fijación intermaxilar), reducción abierta (quirúrgico), tratamiento conservador (fisioterapia y tratamiento funcional con aparatos de ortopedia), el cual es el protocolo que mayormente es utilizado en pacientes pediátricos con resultados satisfactorios a largo plazo. ${ }^{8-10,15}$

El objetivo principal del tratamiento de las fracturas condilares en niños incluye: reducción de los segmentos fracturados, restauración de la oclusión dental, fijación, control de la infección y promoción del remodelado y crecimiento apropiado, minimizar la desviación de la mandíbula, eliminar el dolor, crear un amplio rango de movimientos mandibulares, así como evitar disturbios en el crecimiento y problemas en la ATM. ${ }^{8-15}$ Autores como Dimi- 
troulis, ${ }^{8}$ Defabians, ${ }^{9}$ Peterson, ${ }^{10}$ Medina, ${ }^{14}$ Lobo, ${ }^{16}$ Noleto, ${ }^{17}$ Choi $^{18}{ }^{18}$ Girthofer ${ }^{19}$ apoyan el tratamiento conservador, sugieren que brinda resultados satisfactorios, buena función mandibular, disminución de asimetrías y desviaciones mandibulares, apertura bucal apropiada, excursiones mandibulares aceptables, remodelado condilar y escasas secuelas.

La fisioterapia es una conducta que busca modificar, aliviar o disminuir los hábitos o problemas que causan un impacto negativo en el desarrollo y crecimiento normal del complejo maxilofacial, corrigiendo las relaciones oclusales mediante movimientos de apertura y cierre, así como de lateralidad, mediante la colocación de paletas de madera apiladas o abrebocas colocados con presión para aumentar la apertura bucal de una manera progresiva. $^{20}$

El tratamiento ortopédico temprano en pacientes con fracturas condilares estimula el crecimiento de los tejidos blandos y del cóndilo. La estimulación temprana con el uso de aparatos funcionales y el control de la actividad muscular es un aspecto importante en el tratamiento de las fracturas condilares, la movilización de los tejidos que están dentro y alrededor de la articulación, reducen las cargas de concentraciones de la misma, incrementando el remodelado del cóndilo bajo la propia actividad del sistema masticatorio, previniendo las restricciones mecánicas creadas por cicatrices y la pérdida del movimiento. ${ }^{9}$

El tratamiento conservador permite estimular el remodelado del cóndilo, disminuyendo la posibilidad de asimetrías faciales, acortamiento vertical de la rama mandibular y anquilosis. ${ }^{11,21}$

El objetivo de esta investigación es evaluar una serie de casos de pacientes pediátricos que acuden al servicio de Ortodoncia Interceptiva del Post- grado de Odontopediatría con antecedentes de fractura condilar y así describir las características demográficas de estos pacientes, clasificar el tipo de fractura condilar, describir el tratamiento realizado, comparar la anatomía condilar observada radiográficamente antes y después del tratamiento y comparar las características de simetría facial antes y después de la implementación terapéutica.

\section{Matriales y Métodos}

Esta investigación es de tipo documental, retrospectiva, longitudinal y comparativo (analizando registros de diagnóstico, historias clínicas, fotografías y radiografías previamente recopilados de una serie de casos).

La población son todos los pacientes que acudieron al Servicio de Ortodoncia Interceptiva del Postgrado de Odontopediatría durante el periodo 2001-2014. La muestra se seleccionó a conveniencia y estuvo constituida por todos los pacientes con antecedentes de fracturas condilares que asistieron al Servicio de Ortodoncia Interceptiva del Postgrado de Odontología Infantil durante el periodo comprendido entre 2001-2014.

Fueron incluidos pacientes aparentemente sanos con antecedentes de fracturas condilares, con radiografías panorámicas de buena calidad, en las que se observen claramente ambos cóndilos. Estas radiografías fueron tomadas previamente como parte del diagnóstico integral de los pacientes y no se realizaron tomas específicas para la investigación.

Fueron exluidos mayores de 14 años, niños con síndromes o enfermedades sistémicas, registros diagnósticos incompletos que impidan el diagnóstico de fractura condilar, niños con tratamiento quirúrgico previo o con otras patologías 
de crecimiento mandibular que produzcan asimetrías faciales severas.

Se evaluaron las siguientes variables: fractura condilar (Nivel: cuello del cóndilo alta, media o baja, subcondilar; Número de fracturas: simple, múltiple, conminuta; Posición de la cabeza del cóndilo respecto a la cavidad glenoidea: tallo verde, no desplazada, desplazada, dislocada y situaciones especiales: abierta o cerrada), simetría facial, desviación en apertura.

Se describió el tiempo de seguimiento y el tipo de tratamiento realizado clasificándolo en: Quirúrgico (incluye la reducción abierta) o Conservador (Fijación intermaxilar, Fisioterapia, Ortopedia Funcional de los Maxilares)

Fueron evaluadas las características anatómicas del cóndilo considerando: Anatomía normal del cóndilo, Leve alteración anatómica, Pérdida de la anatomía normal, Anquilosis de la ATM

El resultado terapéutico fue considerado satisfactorio si el paciente aun presentando leve alteración de la anatomía condilar, sigue siendo funcional, no presenta anquilosis y con buenos movimientos excursivos, o, no satisfactorio, si presenta anquilosis, alteraciones de la ATM, dolor.

En este estudio se realizó observación y registro de los datos de las historias clínicas, radiografías y fotografías, a fin de determinar la prevalencia, distribución, consecuencias y tratamiento en cada uno de los pacientes con fractura condilar tratados en el servicio.

La transcripción y tabulación de los datos se realizó en programa Microsoft ${ }^{\circledR}$ Office Excel 2010 (C) Microsoft Corporation, Estados Unidos.
Se realizaron análisis descriptivos: frecuencias, cuadros, gráficos, porcentajes y cuantitativos: media de los valores obtenidos.

Se realizó asesoría y entrenamiento del investigador para el diagnóstico de las fracturas condilares y observación de imágenes.

De cada historia clínica del Servicio de Ortodoncia Interceptiva del postgrado de Odontología Infantil se procedió a tomar los datos demográficos, edad y género, los cuales fueron insertados en la hoja de recolección de datos.

Posteriormente, se evaluó cada radiografía panorámica que estaba en la historia, se evaluaron utilizando negatoscopio, fotografías clínicas, se identificaron las fracturas condilares y se registraron las características como tipo de fractura, tratamiento recibido, tiempo de tratamiento, frecuencia de citas y características radiográficas de la fractura.

Para este proyecto no se recibió financiamiento institucional, siendo totalmente autofinanciado. Los participantes no recibieron remuneración. No hubo conflictos de interés.

Este proyecto recibió el aval del comité de Bioética de la Facultad de Odontología No 0450-2012. El tratamiento que recibieron los pacientes no fue manipulado de forma experimental con objeto de la investigación. Se realiza el seguimiento y la descripción de tratamientos previamente realizados, basados en estándares internacionales de evidencia científica, indicados por un grupo de expertos (asesores) de forma individual ajustada a los requerimientos de cada caso. 


\section{Nivel de la fractura del cóndilo mandibular}

Fractura cuello del cóndilo alta

Fractura cuello del cóndilo baja

Fractura intracapsular

Falta de información

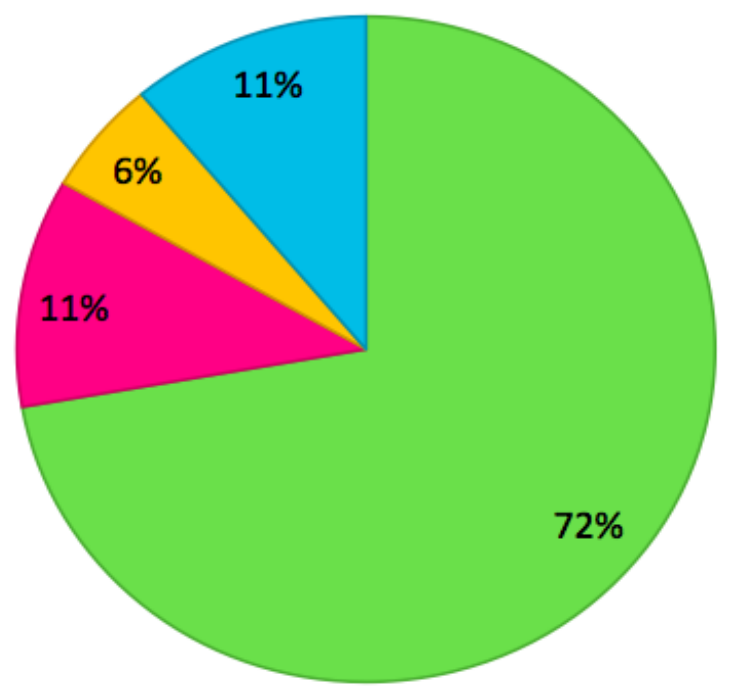

Figura 1. Nivel de la fractura del cóndilo mandibular.

\section{RESULTADOS}

La muestra se seleccionó a conveniencia siguiendo los criterios de inclusión en el período 2001-2014. La población total fue de 867 casos tratados en el servicio de Ortodoncia Interceptiva de 2001 a 2014. De ellos la muestra consistió en 11 pacientes, 5 varones y 6 hembras, representando una prevalencia de $1,50 \%$. La edad promedio que ocurrió el trauma fue de 6.85 años, habiendo recibido pacientes de 1 a 14 años de edad.

Del total de la población fueron excluidos 3 casos por diagnóstico inicial incierto. $\mathrm{Al}$ revisar los registros de pacientes, hubo 3 casos con reporte previo de fractura condilar unilateral a edad muy temprana, pero sin registro inicial que permitiera corroborar la fractura. De estos casos, 2 presentaron anquilosis y 1 asimetría facial severa; habiendo recibido tratamiento quirúrgico fueron ex- cluidos por no poseer registros que permitieran corroborar el diagnóstico de fractura condilar.

Las causas más prevalentes de fractura condilar incluyeron: 7 casos por caídas de altura, 3 casos por accidentes de tránsito y 1 caso por caída de su propia altura.

El total de pacientes fue 11, de ellos 6 presentaron fractura bicondilar y 5 fractura unilateral, siendo que en total fueron evaluados 17 cóndilos con fractura.

Se evaluaron las características de la fractura condilar según el nivel de la fractura, número de fracturas, posición de la cabeza del cóndilo y situaciones especiales. El 100\% de los casos fueron fracturas cerradas del cóndilo. (Figura 1)

En cuanto al número de fractura se incluyeron: 10 casos de fractura simple, 5 casos de fractura 


\section{Condición Anatómica del Cóndilo}

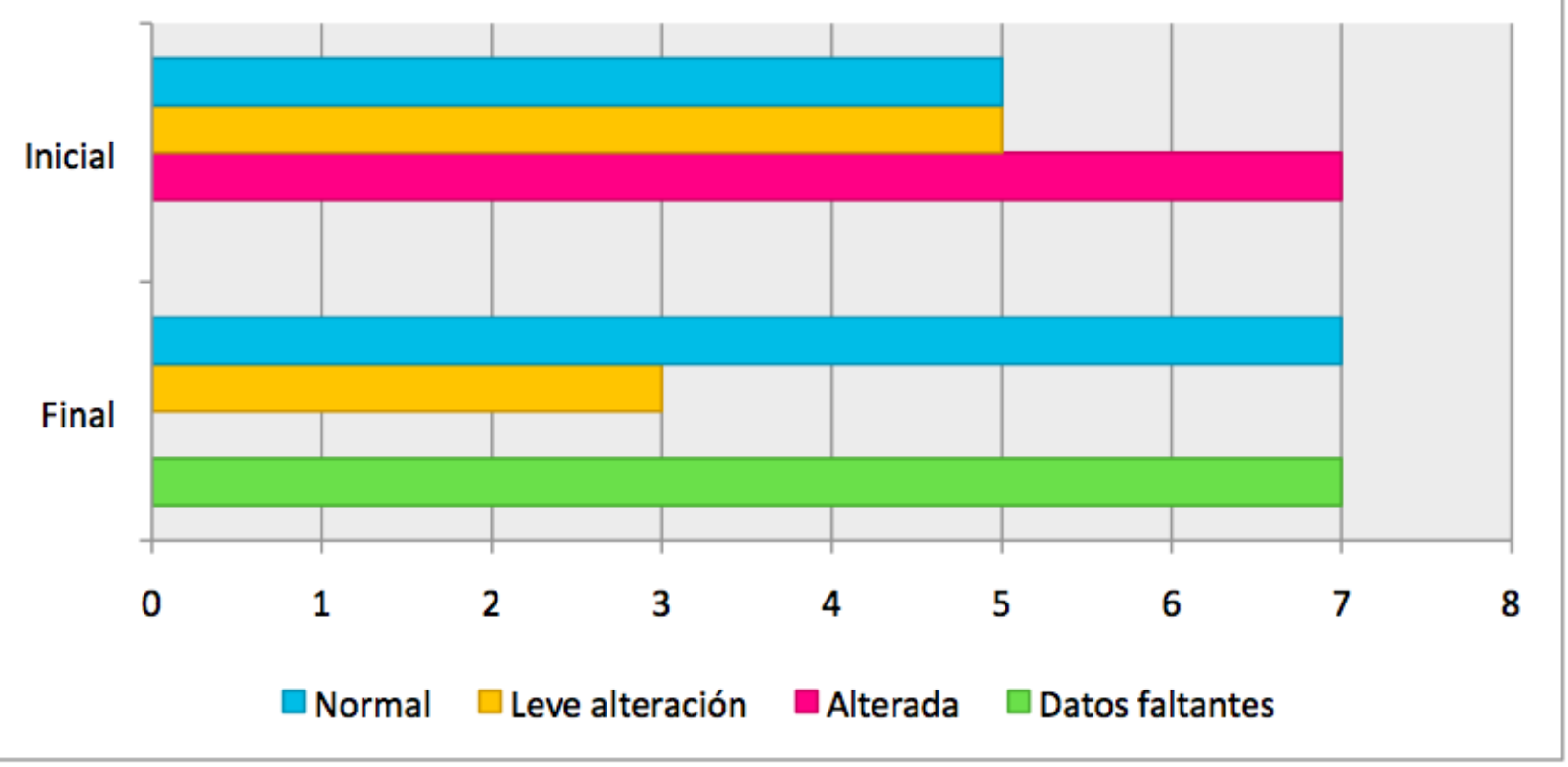

Figura 2. Condición anatómica del cóndilo mandibular, inicial y final.

múltiple, 2 casos donde hubo datos faltantes y no se registró ningún caso de fractura conminuta. El tipo de fractura condilar según la posición de la cabeza del cóndilo con respecto a la cavidad glenoidea incluyeron 8 casos de fracturas dislocadas, 4 casos de fracturas desplazadas, 3 casos de fracturas no desplazadas, ningún caso de fractura en tallo verde y 2 casos donde no hubo registro.

Las características anatómicas del cóndilo mandibular fueron evaluadas a través de radiografías panorámicas, al inicio del tratamiento se encontraron 5 casos con una anatomía normal del cóndilo, 5 casos que presentaron una leve alteración de la anatomía normal, 7 casos con una pérdida de la anatomía normal y no se presentó ningún caso con anquilosis de la ATM en la evaluación inicial. (Figura 2)

Para la última evaluación de las características anatómicas finales del cóndilo se utilizaron las mismas variables que al inicio del tratamiento, los resultados fueron: 7 casos con una anatomía normal, 3 casos presentaron leve alteración de la anatomía y hubo 7 casos donde no se pudo recopilar la información debido a que los pacientes abandonaron el tratamiento. No se registró ningún caso con anquilosis de la ATM. (Figuras 5, 6, 7 y 8)

Para el momento de iniciar el tratamiento, 1 caso presentó asimetría facial, (figura 3) 7 casos presentaron simetría facial y en 3 casos no fueron registrados los datos. En la última evaluación ningún caso reportó asimetría facial, en 5 casos no se pudo recopilar la información. La presencia de desviación en apertura al inicio del tratamiento fue otra variable a ser evaluada en estos casos. Se evaluó si se encontraba presente o ausente. 2 Casos presentaron desviación en apertura, 4 casos no presentaron desviación y hubo 5 casos donde la información no estaba registrada. En la última evaluación se encontraron 5 casos sin desviación 

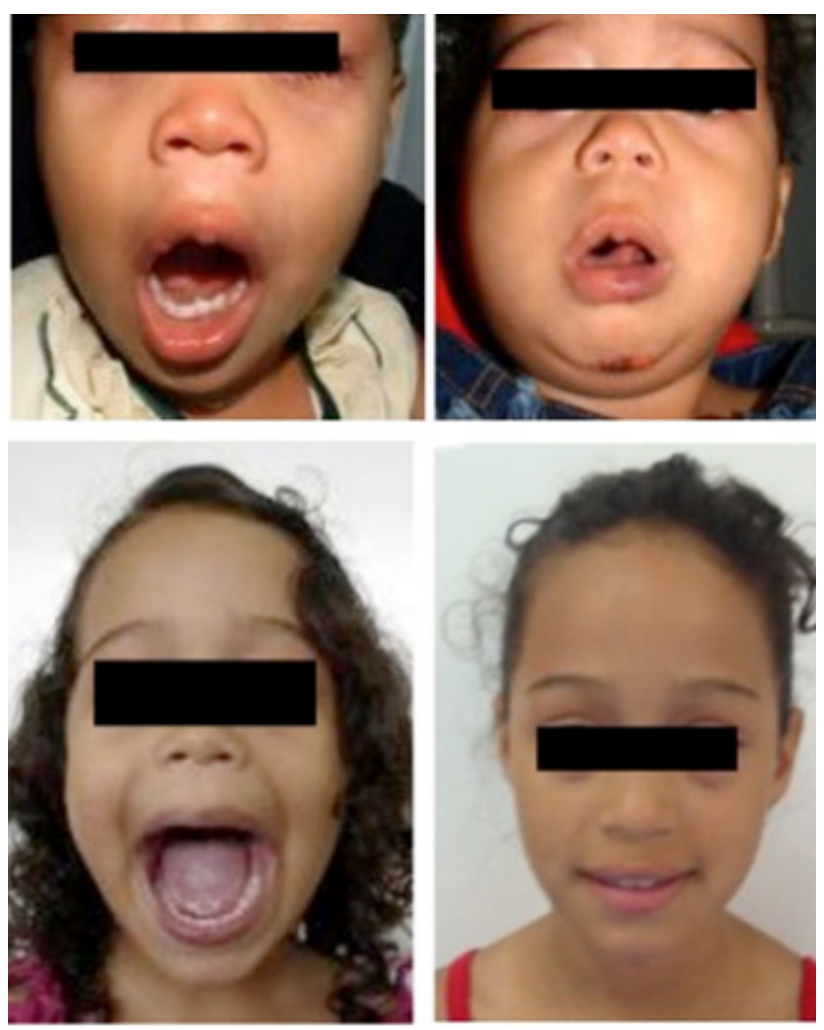

Figura 3. Caso 3: Fotografías extraorales evidenciando asimetría facial y desviación en apertura inicial. En la evaluación a los 5 años se observa corrección en la simetría y en la apertura.

\section{DISCUSIÓN}

El cóndilo mandibular es la estructura más débil del esqueleto facial y la que mayormente puede sufrir consecuencias cuando hay traumatismos en la región facial, produciéndose fracturas del cóndilo mandibular.2-5 De las fracturas en la región facial que ocurren en niños, el cóndilo mandibular es el más afectado debido a su estructura ósea facial, hueso menos compacto, una alta proporción de hueso medular rodeado por una delgada lámina cortical. ${ }^{16,22}$

La prevalencia de las fracturas del cóndilo mandibular es entre $25 \%$ y $35 \%$ del total de las fracturas de los huesos faciales. ${ }^{2}$ En este estudio fue de $1,50 \%$, siendo bastante baja y esto puede ser debido a que este es un servicio de referencia para pacientes que solicitan tratamiento ortodóncico a mediano y largo plazo, no un centro hospitalario donde acuden todos los pacientes en apertura y 6 casos que no se pudo recopilar la información.

La edad promedio de inicio de tratamiento fue de 7 años. El tipo de terapia implementada varió desde fisioterapia, tratamiento con antibioticoterapia y analgesia, terapia conservadora con paletas de mordida y ortopedia funcional y fijación intermaxilar cerrada. En la mayoría de los casos se utilizaron combinaciones de terapia. El tiempo de tratamiento promedio fue de 6 meses, con hasta 2, 6 y 7 años de seguimiento en ciertos casos. (Figura 4)
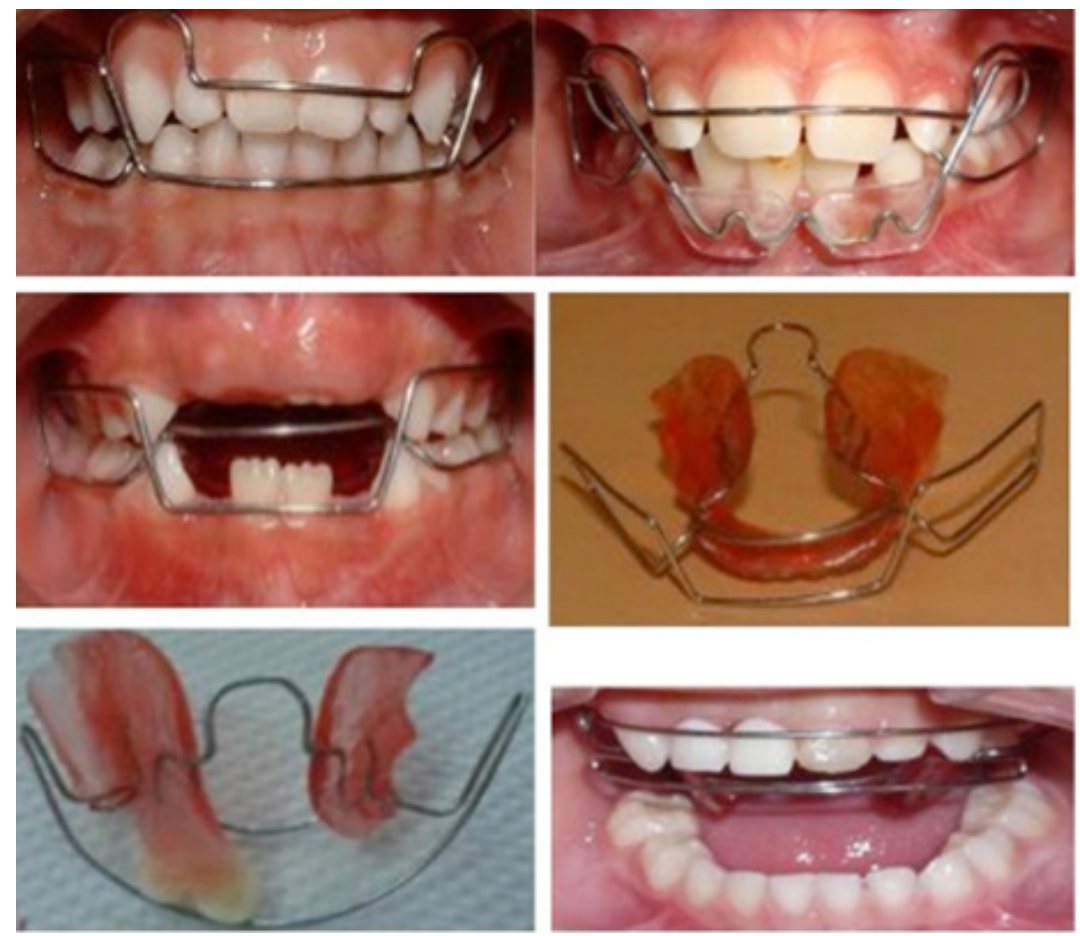

Figura 4. Aparatos de ortopedia funcional utilizados para los diferentes casos, incluyendo Activador de Klammt, Bionator, híbrido Quirós Crespo. 

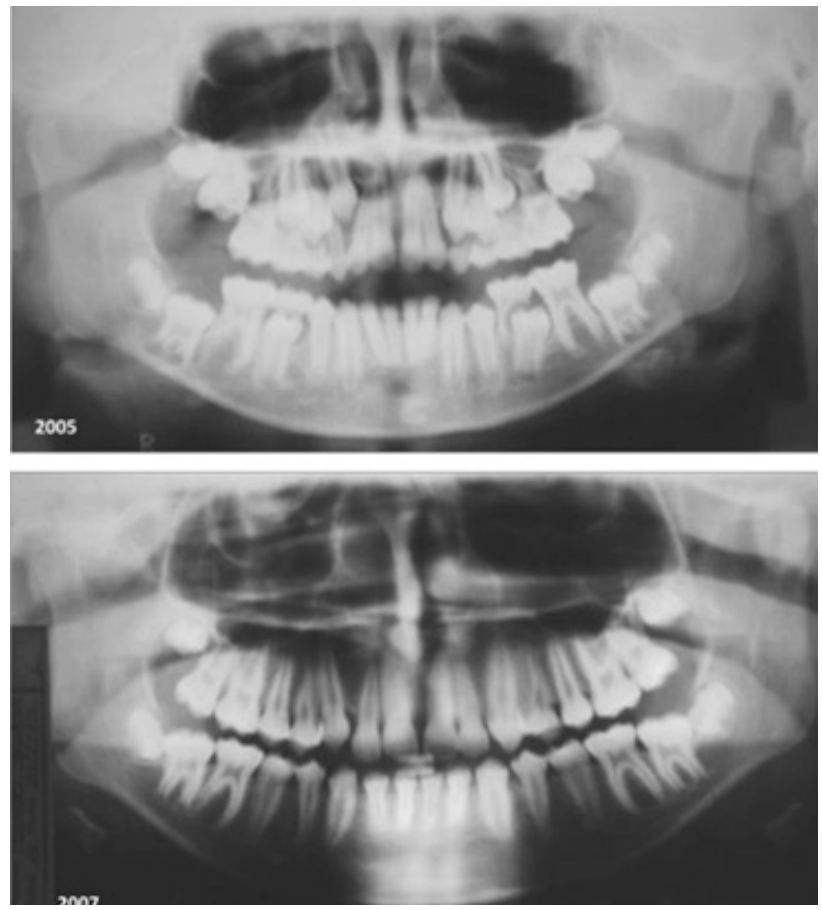

Figura 5. Caso 1: Radiografía panorámica inicial en la que se observa fractura del cuello del cóndilo derecho alta. Radiografía a los dos años de seguimiento con anatomía.

inmediatamente después de un traumatismo para recibir tratamiento de urgencia.

Los estudios indican que los factores etiológicos de las fracturas condilares en niños, van a depender de la edad, la actividad que más desempeñen, la situación socio-económica, situación demográfica, cultural y factores ambientales del paciente. La causa principal de estas fracturas en los niños son las caídas, seguidas por accidentes en bicicleta y accidentes de tránsito. ${ }^{6-8,12,14,16} \mathrm{El}$ maltrato infantil debe considerarse como etiología de las fracturas condilares ya que esto ha cobrado una importancia en la causa de las lesiones del cóndilo en los últimos años. ${ }^{23}$

En los casos aquí reportados se encontró que el factor etiológico predominante con $64 \%$ fueron las caídas de altura en niños menores de 12 años, lo cual coincide con lo descrito en la literatura.
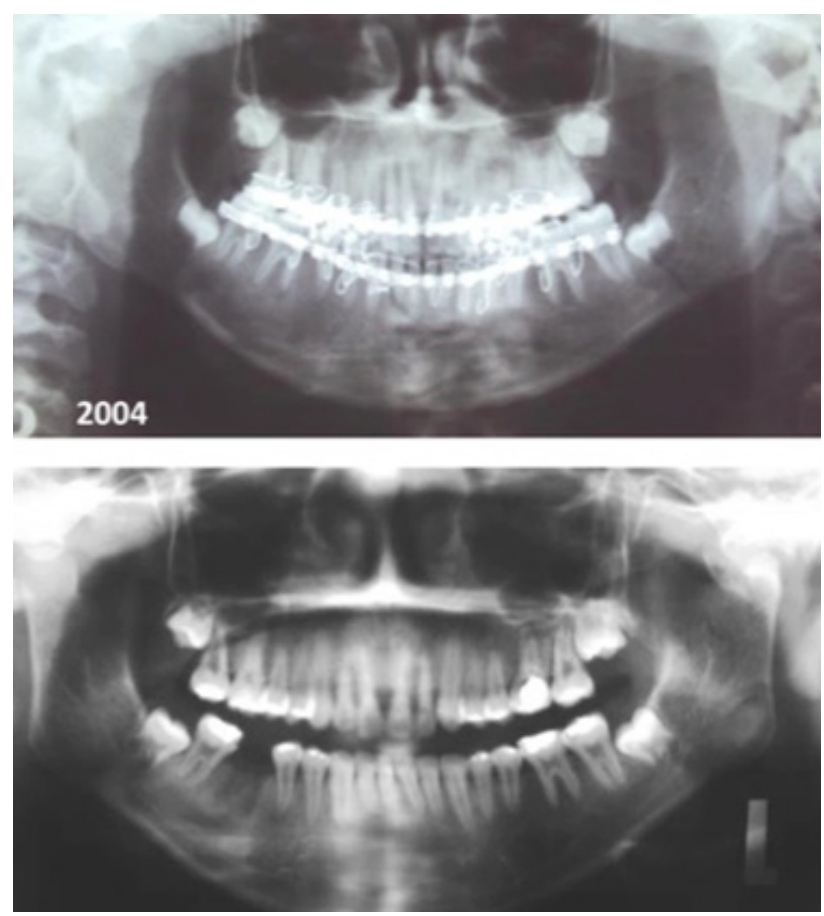

Figura 6. Caso 4: Radiografía panorámica inicial en la que se observa fractura bilateral de los cuellos de los cóndilos, alta desplazada. Radiografía a los 9 meses de seguimiento con leve alteración de la anatomía.

La mayoría de los pacientes presentó caídas de altura, siendo éstas en sus viviendas, de la estructura "platabanda". Esto se refiere a que las condiciones socioeconómicas llevan a estructuras de vivienda con pocas condiciones de seguridad, en las que hay pisos y escaleras sin barandas ni elementos de resguardo que brinden protección de accidentes tipo caídas.

La literatura reporta que la distribución por sexo muestra una prevalencia mayor en niños varones en todos los grupos etarios, aumentando la misma con la edad. ${ }^{6-9,12,15,20}$ En esta serie de casos, la prevalencia fue mayor para niñas.

El Odontopediatra en unión al equipo multidisciplinario, cuenta con una serie de exámenes para establecer un diagnóstico de fractura condilar, evaluando los signos y síntomas de estos pacientes. Uno de los signos característicos que 

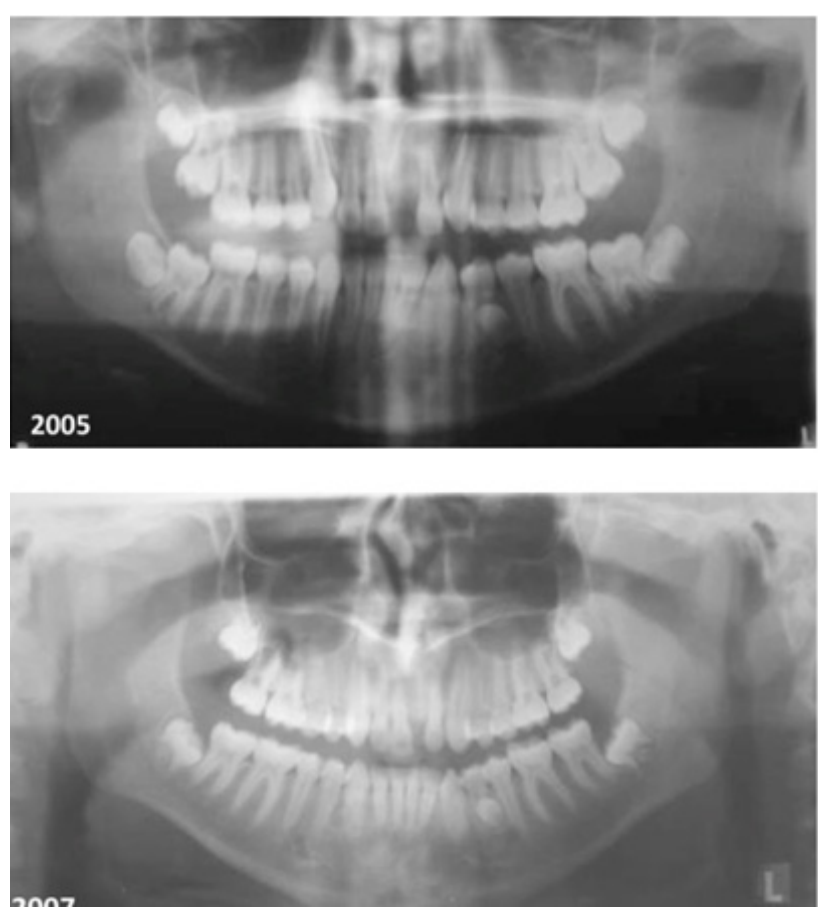

Figura 7. Caso 8: Radiografía panorámica inicial en la que se observa fractura del cuello del cóndilo derecho alta desplazada. Radiografía a los dos años de seguimiento con anatomía normal.

es motivo de sospecha de antecedente de fracturas del cóndilo mandibular es la laceración o contusión del mentón. ${ }^{8,10,32}$

Con respecto al tipo de fractura, muchos sistemas de clasificación han sido propuestos para las fracturas que envuelven el cóndilo mandibular, donde se incluyen: la anatomía, la localización, la línea de fractura, la presencia o ausencia de dientes en la fractura y la exposición de la fractura al medio ambiente..$^{10,28,30}$ Se ha afirmado que en las fracturas del cóndilo en pacientes jóvenes predominan las fracturas intracapsulares y altas del cuello del cóndilo, más que las fracturas subcondilares o bajas que ocurren con una menor proporción. Estas fracturas pueden ocurrir de una manera unilateral o bilateral; siendo las fracturas unilaterales tres veces más frecuentes que las bilaterales. $3,7,10,15,28,32$

En el presente estudio la mayoría de los casos,
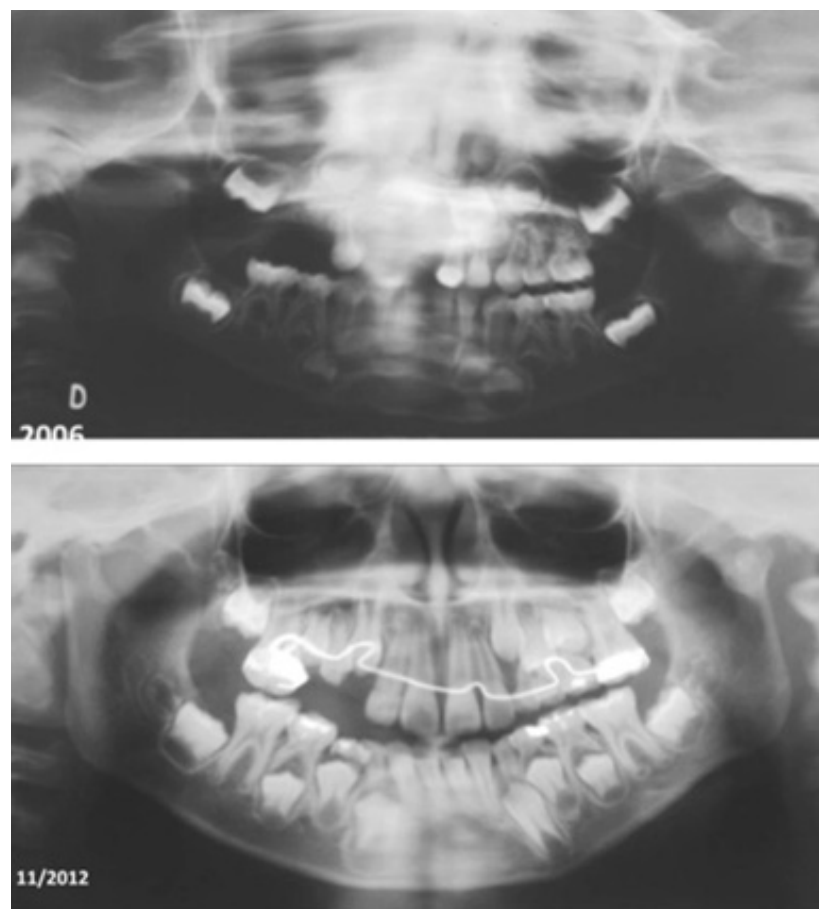

Figura 8. Caso 9: Radiografía panorámica inicial en la que se observa fractura del cuello del cóndilo izquierdo alta dislocada. Radiofrafía a los seis años de seguimiento con alteración en la anatomía

6 fueron fracturas bilaterales, lo que difiere de lo reportado en la literatura. La ubicación más frecuente fue fractura del cuello del cóndilo alta $(76 \%)$, similar a lo reportado por otros autores.

La dislocación del cóndilo de la cavidad glenoidea según ocurre en el 39\% de todas las fracturas condilares pediátricas, dicha dislocación requiere una extensa remodelación para alcanzar una relación anatómica normal. ${ }^{6}$ Particularmente este tipo de fracturas presenta unas características de remodelación incompleta, una formación morfológica anormal en el cuello y en la superficie de la articulación, siendo la asimetría mandibular una consecuencia común en la dislocación del cóndilo. Por lo tanto es de suponer que estas fracturas con dislocación son propensas a producir complicaciones clínicas a largo plazo. ${ }^{6}$

En cuanto al desplazamiento, lo más frecuentemente observado fueron fracturas del cuello 
del cóndilo dislocadas, con un $50 \%$ de prevalencia, y a pesar de que las características anatómicas del cóndilo al inicio del tratamiento fueron con leve alteración o con pérdida de la anatomía normal, estos pacientes con fracturas del cóndilo dislocadas no presentaron asimetría facial lo cual difiere de lo reportado en la literatura por Thorén ${ }^{6}$

El objetivo principal del tratamiento de las fracturas condilares en niños incluye: reducción de los segmentos fracturados, restauración de la oclusión dental, fijación, control de la infección y promoción del remodelado y crecimiento apropiado, minimizar la desviación de la mandíbula, eliminar el dolor, crear un amplio rango de movimientos mandibulares, así como evitar disturbios en el crecimiento y problemas en la ATM. ${ }^{8-15}$

El tratamiento conservador es el más recomen- dado de las fracturas del cóndilo mandibular en pacientes pediátricos. La experiencia ha demostrado buenos resultados en la mayoría de los pacientes después de este procedimiento. Este incluye fisioterapia con ejercicios de apertura y cierre con paletas baja lenguas apiladas juntas, para aumentar la apertura bucal y el uso de aparatos funcionales que ayudan a mantener la mandíbula en una posición adecuada, estimulando sus funciones y guiando el crecimiento. El tratamiento dependerá de la edad, de la extensión del daño y del momento en que se diagnos-

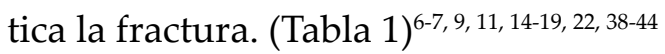

La fijación intermaxilar consiste en la restauración normal de la oclusión mediante la inmovilización de la mandíbula por medio de fijación intermaxilar con elásticos o ligaduras metálicas. De ser necesario es seguida por fisioterapia, o con el uso de aparatos de ortopedia funcional. ${ }^{11}$, 18, 21, 30 En el presente estudio de 11 casos presen-

\begin{tabular}{|c|c|c|c|c|c|c|c|c|}
\hline Autor & Año & Tipo & $\operatorname{Sin} \mathrm{Tx}$ & FIM & Fisioterapia & OFM & Cirugía & Resultado \\
\hline Hotz $^{2}$ & 1978 & 2 casos & & & & $\mathrm{x}$ & & Satisfactorio \\
\hline Amaratunga $^{7}$ & 1992 & 17 casos & & $\mathrm{x}$ & & & & Satisfactorio \\
\hline Kahl y Cols. ${ }^{41}$ & 1995 & 19 casos & & & & $\mathrm{x}$ & & Satisfactorio \\
\hline Guven $^{11}$ & 2001 & 18 casos & & $\mathrm{x}$ & $\mathrm{x}$ & & & Satisfactorio \\
\hline Thorén y Cols. ${ }^{6}$ & 2001 & 18 casos & & $\mathrm{x}$ & $\mathrm{x}$ & & & Satisfactorio \\
\hline Defabianis $^{15}$ & 2001 & 2 casos & & & & $\mathrm{x}$ & & Satisfactorio \\
\hline Ramírez y Cols. ${ }^{43}$ & 2002 & 1 caso & $\mathrm{x}$ & & & & & Satisfactorio \\
\hline Girthofer y Cols. ${ }^{19}$ & 2002 & 1 caso & & $\mathrm{x}$ & & $\mathrm{x}$ & & Satisfactorio \\
\hline Defabianis $^{9}$ & 2003 & 25 casos & & & $\mathrm{x}$ & $\mathrm{x}$ & & Satisfactorio \\
\hline Schoen y Cols. ${ }^{38}$ & 2005 & 1 caso & & & & & $\mathrm{x}$ & Satisfactorio \\
\hline Choi y Cols. ${ }^{18}$ & 2005 & 11 casos & & $\mathrm{x}$ & $\mathrm{x}$ & & & Satisfactorio \\
\hline Deleyainnis y Cols. ${ }^{39}$ & 2006 & 6 casos & & & & & $\mathrm{x}$ & No Satisfactorio \\
\hline Landes y Cols. ${ }^{44}$ & 2008 & 24 casos & & $\mathrm{x}$ & & & $\mathrm{x}$ & Satisfactorio \\
\hline Medina $^{14}$ & 2009 & 1 caso & & & $\mathrm{x}$ & $\mathrm{x}$ & & Satisfactorio \\
\hline Noleto y Cols. ${ }^{17}$ & 2011 & 1 caso & & $\mathrm{x}$ & $x$ & & & Satisfactorio \\
\hline Lobo y Cols. ${ }^{16}$ & 2011 & 3 casos & & & $\mathrm{x}$ & $\mathrm{x}$ & & Satisfactorio \\
\hline Leuin y Cols. ${ }^{22}$ & 2011 & 83 casos & & $\mathrm{x}$ & & & $\mathrm{X}(1)$ & Satisfactorio \\
\hline Tuna y Cols. ${ }^{40}$ & 2012 & 1 caso & & & $\mathrm{x}$ & $\bar{x}$ & & Satisfactorio \\
\hline
\end{tabular}

Tabla 1. Resumen de la revisión de la literatura. 
tados, sólo un caso fue tratado con fijación intermaxilar seguido por fisioterapia y tratamiento con aparato funcional, con buena evolución del caso, lo que coincide con lo reportado en la literatura.

En el presente estudio 7 casos recibieron tratamiento conservador con paletas de mordida obteniendo buenos resultados funcionales, lo que coincide con lo reportado. , 8-10,36 $^{-1}$

La selección del tipo de aparato funcional va a depender principalmente del tipo de fractura que presente el paciente, la gravedad del mismo, el diagnóstico dentario que éste presente y de la tendencia al crecimiento dado por el estudio cefalométrico realizado al paciente. ${ }^{14}$ Esta serie de casos reportados coincide con los resultados obtenidos en la literatura por quienes trataron la mayoría de los casos con aparatos de ortopedia funcional, cursando con una evolución favorable, es decir, los pacientes luego del tratamiento no presentaron asimetrías, anquilosis, desviaciones en apertura, problemas articulares, entre otros; aunque se observaron variaciones anatómicas en el remodelado condilar. 3, 6-10,14, 16, 17, 19, 40-42

En niños menores de 12 años de edad, el tratamiento conservador es posible dada la alta capacidad de regeneración morfológica y funcional que presenta el cóndilo. Investigaciones han demostrado la capacidad regeneradora del proceso condilar especialmente en pacientes en crecimiento, evidenciando que el potencial de cicatrización y remodelamiento óseo es mayor en los niños que en los adultos. ${ }^{6-8}$

A pesar de que la literatura sostiene que en niños mayores de 12-14 años el tratamiento debe ser quirúrgico, como en adultos, debido a que se espera pobre remodelado del cóndilo, en el presen- te estudio, un caso de paciente masculino de 12 años de edad, con fractura dislocada se observó remodelado condilar óptimo. Así mismo se presentaron 2 casos de pacientes en crecimiento con fractura condilar desplazada y dislocada, luego de recibir tratamiento con fisioterapia y aparatos de ortopedia funcional, se pudo evidenciar en las radiografías control remodelado condilar.

Los pacientes con fractura del cuello del cóndilo alta, entre 1 y 3 años de edad, que presentaron asimetría facial, recibieron tratamiento con aparatos funcionales, Quirós-Crespo y Klammt respectivamente. Tomando en consideración que estos iniciaron tratamiento 2 y 8 meses después de la fractura, aun así la evolución de los casos fue muy favorable con un seguimiento de 7 años.

En cuanto al diagnóstico se considera que los estudios radiográficos son una herramienta necesaria para completar el examen clínico. Peterson $^{10}$ y Dimitroulis ${ }^{8}$, estudiaron que la radiografía panorámica es comúnmente el estudio más accesible para los odontólogos y constituye una excelente base para el diagnóstico de las fracturas. Ellis en 2000 comenta que es una técnica que aporta información en cuanto a la localización y la existencia o no de desplazamiento condilar, pero que no es lo suficientemente resolutiva. Es por ello que ha sido reemplazada, casi completamente, por la tomografía computarizada. ${ }^{45}$

En los casos reportados la técnica radiográfica empleada para el diagnóstico de fracturas condilares fue radiografía panorámica, sólo uno de los casos se presentó al servicio con tomografía axial computarizada que ya había sido indicada en la emergencia del centro hospitalario que éste acudió. Lamentablemente en los servicios de salud pública, los médicos y odontólogos que reciben a estos pacientes no indican una tomografía axial computarizada de 
rutina, debido a su alto costo, no obstante debería considerarse dentro de los exámenes de rutina en los pacientes con fracturas condilares, para el mejor diagnóstico tridimensional de la afección.

La anquilosis de la articulación temporomandibular es una complicación común en las fracturas condilares bilaterales. La consolidación espontánea de una fractura condilar puede conducir a una anquilosis funcional, lo que va a dar lugar a una deformidad mandibular y a una alteración de las estructuras relacionadas. Es posible que la anquilosis afecte el crecimiento, a pesar de que la mandíbula sea capaz de moverse, esta presenta una restricción para desplazarse hacia adelante fuera de la fosa glenoidea presentando así, limitaciones funcionales en el movimiento. ${ }^{15,17}$

En el presente estudio ninguno de los casos reportados, independientemente de la edad del paciente, del tipo de fractura y del tratamiento recibido, presentaron anquilosis, lo cual es de gran importancia ya que esta es una de las mayores y más graves complicaciones observadas en este tipo de traumas.

El seguimiento de estos pacientes es fundamental, deben ser controlados clínica y radiográficamente no solamente durante su periodo de cicatrización, sino por un largo tiempo hasta que el crecimiento facial haya terminado y la oclusión dental permanente sea estable. Esto es con la finalidad de que, si se presenta alguna alteración durante este periodo, pueda ser tratada y canalizada antes de que se produzcan complicaciones.

Varios de los casos reportados en este estudio presentaron registros iniciales o finales incompletos, haciendo imposible la comparación y seguimiento. Las citas fueron registradas de forma poco escrita. (Tabla 2) Se recomienda la estandarización en el registro de datos y seguimiento de futuros casos de los pacientes que acudan al servicio con antecedentes de fractura del cóndilo mandibular.

El manejo multidisciplinario de los pacientes con fractura del cóndilo es clave, el equipo formado por el cirujano bucal y maxilofacial y odontopediatra va a permitir que la evolución del paciente pediátrico con fracturas del cóndilo mandibular pueda realizarse de una manera más oportuna y eficiente durante su crecimiento y desarrollo facial.

\section{CONCLUSIONES}

Las fracturas del cóndilo mandibular son poco frecuentes en la población que fue estudiada, representando el 1,50\% de los casos. La edad promedio en la que ocurrió el trauma fue de 6 años. Hubo predilección por el sexo femenino.

Las causas más frecuentes en esta muestra con fractura condilar fueron caídas de altura (64\%) y accidentes de tránsito (27\%).

El tipo de fractura que prevaleció fue fractura condilar unilateral en 6 de los casos. Según el número, la mayoría de las fracturas reportadas fueron simples y con respecto a la posición de la cabeza del cóndilo con respecto a la cavidad glenoidea las fracturas dislocadas tuvieron mayor prevalencia.

El tratamiento conservador, incluyendo fisioterapia y ortopedia funcional de los maxilares, resultó exitoso en la mayoría de los casos. Como resultado terapéutico se observó remodelado del cóndilo, en ocasiones con leves variantes anatómicas, simetría facial en reposo y apertura. Ningún caso presentó anquilosis de la ATM. 


\begin{tabular}{|c|c|c|c|c|c|c|c|c|c|c|c|c|c|c|c|c|c|c|}
\hline 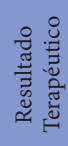 & & 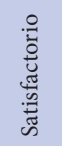 & 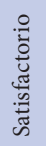 & 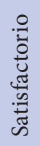 & 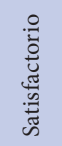 & 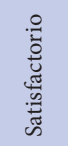 & 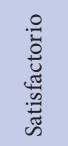 & 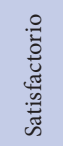 & 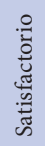 & 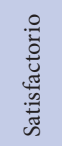 & 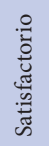 & 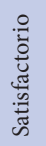 & 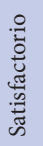 & 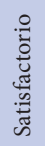 & 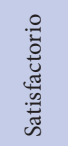 & 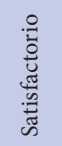 & 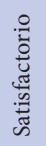 & 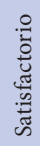 \\
\hline \multirow{2}{*}{ 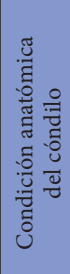 } & 茎 & 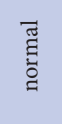 & 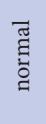 & $\begin{array}{l}\text { בే } \\
\text { हू } \\
\Xi\end{array}$ & 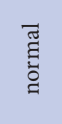 & 芯 & 总 & * & * & * & * & 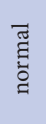 & 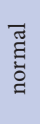 & 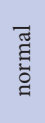 & 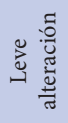 & * & * & $\star$ \\
\hline & $\begin{array}{l}\bar{J} \\
. \mathbb{\Xi} \\
]\end{array}$ & 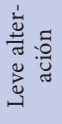 & 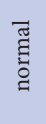 & 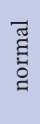 & 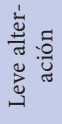 & 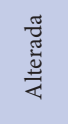 & 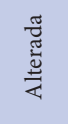 & 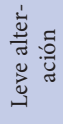 & 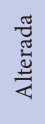 & 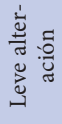 & $\begin{array}{l}\bar{\Xi} \\
\text { हี } \\
\square\end{array}$ & $\begin{array}{l}\bar{\Xi} \\
\stackrel{\Xi}{0} \\
\stackrel{\Xi}{a}\end{array}$ & 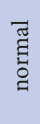 & $\begin{array}{l}\frac{\pi}{\tilde{J}} \\
\frac{\tilde{U}}{4}\end{array}$ & 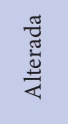 & 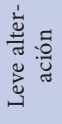 & 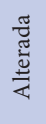 & 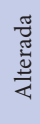 \\
\hline
\end{tabular}

\begin{tabular}{|c|c|c|c|c|c|c|c|c|c|c|c|c|c|c|c|c|c|c|}
\hline $\begin{array}{l} \pm \\
\pm \\
\approx\end{array}$ & 茎 & * & $\stackrel{\circ}{g}$ & 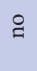 & $\stackrel{8}{8}$ & * & * & * & * & ̊ & 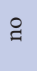 & * & * & * & * & $\stackrel{8}{\AA}$ & $\stackrel{\circ}{\exists}$ & 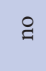 \\
\hline 它 & $\begin{array}{l}\bar{J} \\
\stackrel{\Xi}{\Xi} \\
\Xi\end{array}$ & * & g & ‡ & $\bar{\omega}$ & * & * & * & * & ஜ & \& & ‡ & ‡ & * & * & $\bar{n}$ & ̊ & ‡ \\
\hline
\end{tabular}

\begin{tabular}{|c|c|c|c|c|c|c|c|c|c|c|c|c|c|c|c|c|c|c|}
\hline & 茎 & * & क्ष & "w & $\bar{\omega}$ & * & * & "w & $\bar{\omega}$ & के & $\bar{\infty}$ & * & * & * & क & * & "s & $\bar{\omega}$ \\
\hline हैं & 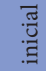 & * & $\bar{\omega}$ & $\bar{\omega}$ & ̊ & * & * & $\bar{\omega}$ & $\bar{\omega}$ & $\bar{s}$ & $\bar{n}$ & $\bar{w}$ & $\bar{w}$ & $\bar{w}$ & $\bar{\omega}$ & * & $\bar{n}$ & $\bar{\omega}$ \\
\hline
\end{tabular}

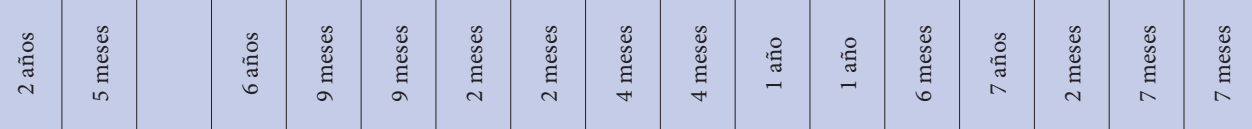

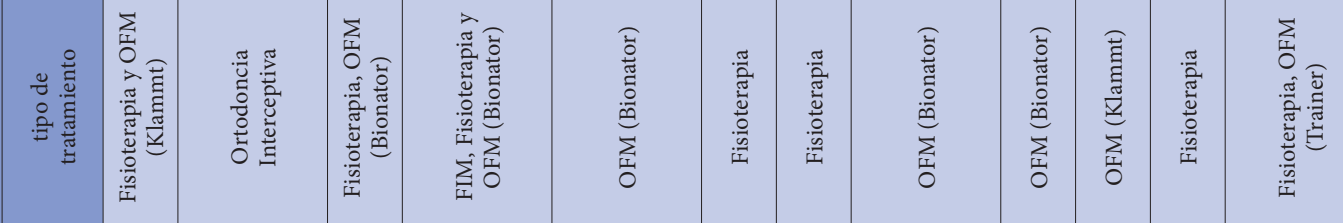

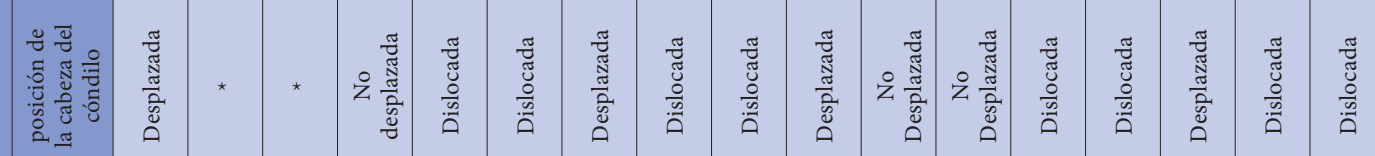

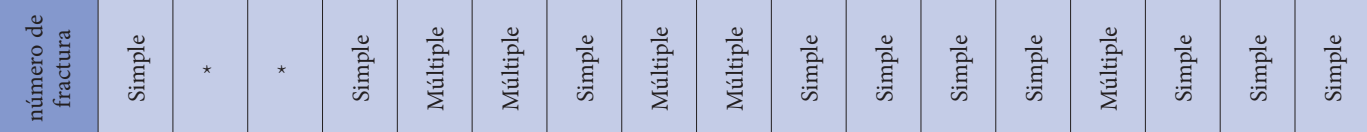

\begin{tabular}{|c|c|c|c|c|c|c|c|c|c|c|c|c|c|c|c|c|c|}
\hline 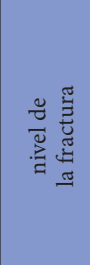 & 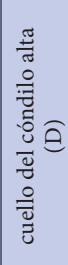 & 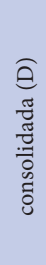 & 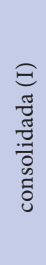 & 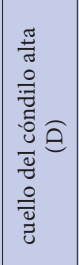 & 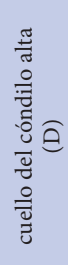 & 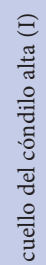 & 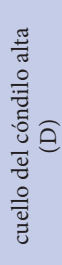 & 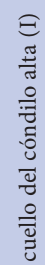 & 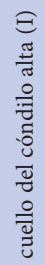 & 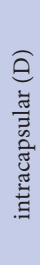 & 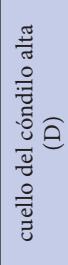 & 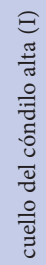 & 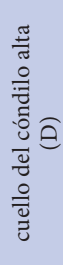 & 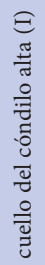 & 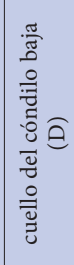 & 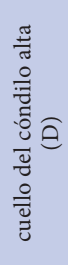 & ت \\
\hline
\end{tabular}

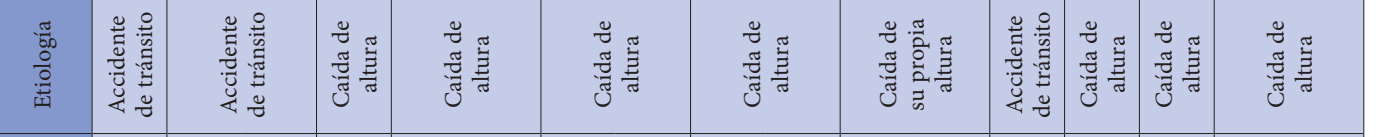

\begin{tabular}{|c|c|c|c|c|c|c|c|c|c|c|c|}
\hline $\begin{array}{l}\vec{J} \\
\text { ] }\end{array}$ & 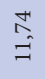 & $\begin{array}{l}\bar{\sigma} \\
\infty\end{array}$ & $\stackrel{\infty}{\stackrel{\infty}{\sim}}$ & $\begin{array}{l}\text { D } \\
\stackrel{-}{-}\end{array}$ & $\begin{array}{l}\infty \\
0 \\
\infty\end{array}$ & $\stackrel{\circ}{=}$ & $\hat{o}_{\infty}^{\infty}$ & $\begin{array}{l}\infty \\
\stackrel{\infty}{=}\end{array}$ & 今े & $\begin{array}{c}\tilde{N} \\
\tilde{m}\end{array}$ & in \\
\hline 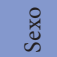 & $\Sigma$ & 山 & 山 & 工 & 山 & $\Sigma$ & $\Sigma$ & $\Sigma$ & $\Sigma$ & 山 & 山 \\
\hline $\begin{array}{l}8 \\
\text { \&ू } \\
\tilde{\Xi}\end{array}$ & - & $N$ & $m$ & $r$ & in & 0 & n & $\infty$ & $a$ & 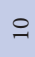 & $=$ \\
\hline
\end{tabular}




\section{Referencias bibliográficas}

1. Throckmorton G, Talwar R, Ellis E. Changes in masticatory patterns after bilateral fracture of the mandibular condylar process. J Oral Maxillofac Surg. 1999 May; 57(5): 500-8.

2. Valiati R, Ibrahim D, Abreu ME, Heitz C, Oliveira RB, Pagnoncelli RM, et al. The treatment of condylar fractures: to open or not to open? A critical review of this controversy. Int. J. Med. Sci. 2008; 6: 313-318.

3. Marker P, Nielsen A, Bastian HL. Fracture of mandibular condyle. Part 1: Patterns of distribution of types and causes of fractures in 348 patients. Br J Oral Maxilofac Surg. 2000; 38: 417-21.

4. Fasola AO, Obiechina AE, Arotiba JT. Incidence and pattern of maxillofacial fractures in the elderly. Int J Oral Maxillofac Surg. 2003;(32): 206-11.

5. Ellis E, Moos KF. Ten years of mandibular fractures: An analysis of 2,137 cases. Oral Surg Oral Med Oral Pathol. 1985; 59: 120-29.

6. Thoren H, Lizuka T, Hallikainen D, Nurminen M, Lindqvist C. An epidemiological study of patterns of condylar fractures in children. Br J Oral Maxillofac Surg. 1997; 35(5): 306-11.

7. Amaratunga NA. Mandibular fractures in Sri Lankan children: a study of clinical aspects, treatment needs and complications. J Dent Children. 1992; 59(21): 111- 4.

8. Dimitroulis G. Condylar injuries in growing patients. Aust. Dent. J. 1997; 42(6): 367-71.

9. Defabians P. TMJ fractures in children and adolescents: treatment guidelines. J Clin Pediatr Dent. 2003; 23(3): 191-9.

10. LJ P. Principles of oral and maxillofacial surgery: Editorial Lippincott Willians and Wilkins; 1992.

11. Guven O, Keiskin A. Remodeling following condylar fractures in children. J. Maxillofac. Surg. 2001; 29: 232-7.

12. Atilgan S, Erol B, Yaman F, Yilmaz N, Ucan MC. Mandibular fractures: a comparative analysis between young and adult patients in the southeast region of Turkey. J. Appl. Oral Sci. 2009; 18(1): 17-22.

13. Alcala-Galiano A, Arribas-Garcia I, Martin-Perez MA, Romance A, Montalvo-Moreno JJ, Millan JM.

Pediatric facial fractures: children are not just small adults. Radiograp. 2008; 28: 441-61.

14. Medina AC. Functional Appliance Treatment for Bilateral Condylar Fracture in a Pediatric Patient. Pediatr Dent. 2009 Sep; 31(5): 432-7.

15. DeFabianis P. TMJ fractures in children: clinical management and folow up. J Clin Pediatr Dent. 2001; 25(3): 203-8.

16. Lobo C, Medina AC, Crespo O. Tratamiento funcional conservador en las fracturas condilares en niños. Reporte de tres casos. MedULA. 2011; 20(1): 76- 86.

17. Noleto JW, Leao EI, Braga CL, Yang S, Sardow A. Conservative approach of condylar fracture in a child by the use of rubber elastics: 7 year follow up. J. Dent. Child. 2011; 78(3): 148-53.

18. Choi J, Oh N, Kim IK. A follow-up study of condyle fracture in children. Int. J. Oral Maxillofac. Surg. 2005; 34: 851-8.

19. Girthofer K, Goz G. TMJ remodeling after condylar fracture and functional jaw orthopedics a case report. J. Orofac Orthop. 2002; 63(5): 429-34.

20. Crean ST, Sivarajasingam V, Fardy MJ. Conservative approach in the management of mandibular fractures in the early dentition phase. A case report and review of the literature. Int J Paediatr Dent. 2000; 10: 291-6. 
21. Zimmermann CE, Troulis MJ, Kaban LB. Pediatric facial fractures: recent advances in prevention, diagnosis and management. Int J Oral Maxillofac Surg. 2006; 35 (1): 2-13.

22. Leuin SC, Frydenball E, Gao D, Chan KH. Temporomandibular Joint Dysfunction After Mandibular Fracture in Children. Arch Otolaryngol Head Neck Surg. 2011; 137(1): 10-4.

23. Galiano AA, García IA, Pérez MM, Romance A, Moreno JM, Millán Funcos JM. Pediatric Facial Fractures: Children Are Not Just Small Adults. RadioGraph. 2008; 28 (2): 441-461.

24. Zix JA, Schaller B, Lieger O, Saulacic N, Thorén H, Lizuka T. Incidence, aetiology and pattern of mandibular fractures in central Switzerland. Euro. J. Med. Scien. 2011; 141: p. w13207.

25. Lindahl L, Hollender L. Condylar fractures of the mandible II. A radiographic study of remodeling processes in the temporomandibular joint. Int Oral Surg. 1977; 6(3): 153-65.

26. Defabianis P. The importance of Early Recognition of Condylar Fractures in Children: A Study of 2 Cases. J. Orofac. Pain. 2004; 18(3).

27. Putz R, Pabst R. Atlas de anatomia humana de sobotta. 21st ed.: Medica Panamericana; 2001.

28. Lindahl L. Condylar fractures of the mandible. I. Classification and relation to age, occlusion, and concomitant injuries of teeth and teeth-supporting structures, and fractures of the mandibular body. Int J Oral Surg. 1977; 6(1): 12-21.

29. Sanders B. Cirugia bucal y maxilofacial pediatrica Buenos Aires: Mundi; 1984.

30. Garcia D, Masia A, Pons G. Sociedad Española de Cirugía Plástica, Reparadora y Estética. [Online].; 2000-2001 [citado 2005 Junio 8. Disponible en: http://www.secpre.org/documentos\%20manual\%2040b.html.

31. Cho BH. Diagnostic performance of dental students in identifyng mandibular condyle fractures by panoramic radiography and the usefulness of reference images. Korean Acad. O. Maxillofac. Radio. 2011; 41: 53-7.

32. Santler G, Karcher H, Ruda C, Kole E. Fractures of the condylar process: surgical versus nonsurgical treatment. J Oral Maxillofac Surg. 1999; 57(4): 392-7.

33. Haug RH, Foss J. Maxillofacial injuries in the pediatric patient. Oral Surg Oral Med Oral Pathol Radiol Endod. 2000; 90(2): 126-34.

34. Defabians P. Treatment of condylar fractures in children and youths: the clinical value of the occlusal plane orientation and correlation with facial development (case report). J. Clin. Pediat. Dent. 2002; 26(3): 243-50.

35. Sandner O. Tratado de cirugía oral y maxilofacial Caracas: Amolca; 2007.

36. Morgan DH, House LR, Hall WH, Vamvas SJ. Diseases of the Temporomandibular Apparatus: A Multidisciplinary Approach. Morby Publishers. 1982 May; 8(23): 156-7.

37. Norholt SE, Krishnan V, Sindet-Pedersen S, Jensen I. Pediatric condylar fractures: a long-term follow-up study of 55 patients. J. O. Maxillofac. Surg. 1993; 51(12): 1302-10.

38. Schoen R, Gellrich NC, Schmelzeisen R. Minimally invasive open reduction of a displaced condylar fracture in a child. Brit J O. Maxillofac. Surg. 2005; 43: 258- 60.

39. Deleyiannis FW, Vecchione L, Martin B, Jiang S, Sotereanos G. Open reduction and internal fixation of dislocated condylar fractures in children: long-term clinical and radiologic outcomes. Ann Plast Surg. 2006; 57(5): 495-501.

40. Tuna EB, Dundar A, Cankaya AB, Gencay K. Conservative Approach to Unilateral Condylar Fracture in a Gorwing Patient: 2.5 Year Follow Up. Open Dent. J. 2012; 6: 1-4.

41. Kahl B, Fischbach R, Gerlach KL. Temporomandibular joint morphology in children after treatment of condylar fractures with functional appliance therapy: a follow-up study using spiral computed tomography. Dentomaxillofac. Radiol. 1995; 24: 37- 45. 
42. Hotz RP. Functional jaw orthopedics in the treatment of condylar fractures. Amer. J. Orthodont. 1978 Abril; 73(4): 365-77.

43. Ramirez-Yañez GO, Young WG. Condylar Fracture: Nontreatment Case Followed Over 23 Years. W. J. Orthodont. 2002; 3(4): 349-52.

44. Landes CA, Lipphardt R. Prospective evaluation of a pragmatic treatment rationale: open reduction and internal fixation of displaced and dislocated condyle and condylar head fractures and closed reduction of nondisplaced, non-dislocated fractures. Int. J. Oral Maxillofac. Surg. 2006; 35: 115-26.

Recibido: 20/11/2016

Aceptado: 12 /01/2017

Correspondencia: Ana Verónica D'Andrea anavero64@hotmail.com 